\title{
RESONANCE-BASED NORMALIZATION THEORY FOR ANALYSIS AND DESIGN OF RESONANT POWER CONVERTERS
}

\author{
Lucas S. Mendonça ${ }^{1,2}$; Fábio E. Bisogno ${ }^{2}$ \\ ${ }^{1}$ Fraunhofer IIS/EAS, Dresden - Saxony, Germany \\ ${ }^{2}$ Universidade Federal de Santa Maria (UFSM) - Santa Maria - RS, Brazil \\ e-mail: lucassangoi1993@gmail.com
}

\begin{abstract}
This paper deals with a circuit modeling approach based on a normalization procedure in order to represent the system considering resonant parameters instead of real circuit parameters. The main advantages are the attainment of generalized solutions that do not depend on specifications, which allows the design of the converter without resorting to circuit level simulations. In this work, the fundamentals and the mathematical formulation of the normalized analysis are described. Furthermore, a multi-stage Class D-E DCDC resonant converter is analyzed without simplifying assumptions used in other methodologies, such as, high loaded quality factor and fundamental component approximation. Experimental results are shown to verify the theoretical approach.
\end{abstract}

Keywords - Circuit Analysis, DC-DC Converters, Normalized Analysis, Resonant Power Conversion, StateSpace Methods.

\section{NOMENCLATURE}

$\begin{array}{ll}\lambda & \text { Index for the converter operating mode. } \\ a & \text { Inverse transfer power ratio. } \\ A_{j} & \text { Normalized resonant frequency. } \\ D_{c} & \text { Duty cycle. } \\ \mathbf{E}_{\lambda} & \text { State matrix. } \\ \mathbf{F}_{\lambda} & \text { Input matrix. } \\ \mathbf{G}_{\lambda} & \text { Output matrix. } \\ \mathbf{H}_{\lambda} & \text { Transmission matrix. } \\ j, k & \text { Indexes to define number resonant parameters. } \\ l, m, n & \text { Indexes to define number of components. } \\ n_{A_{j}} & \text { Number of normalized resonant frequencies. } \\ n_{Q_{k}} & \text { Number of quality factors. } \\ n_{v r} & \text { Number of resulting variables. } \\ \mathbf{P} & \text { Equivalence transformation. } \\ Q_{k} & \text { Quality factor. } \\ t_{\lambda} & \text { Time that ends operating mode. } \\ T_{\lambda} & \text { Duration of operating mode. } \\ T_{P O T} & \text { Transfer power ratio. } \\ \omega_{j} & \text { Angular frequency. } \\ \mathbf{x}(t) & \text { Vector space. } \\ \mathbf{x}_{\mathbf{e}}(\omega t) & \text { Vector space. } \\ \mathbf{y} & \text { Output vector. }\end{array}$

"Manuscript received 05/03/2019; first revision 24/04/2019; accepted for publication 18/07/2019, by recommendation of Editor Marcello Mezaroba. http://dx.doi.org/10.18618/REP.2019.3.0018

\section{INTRODUCTION}

High efficiency power conversion systems are required in several applications, such as, wireless power transfer [1][4], energy harvesting [5]-[7] and artificial lighting [8]-[11]. Miniaturization, high frequency operation and high efficiency are desired features in power conversion electronics [12]. However, to acquire improved performance, an adequate and well-defined methodology is mandatory, especially when resonant power conversion is used, which requires a good balance among complexity, accuracy and time-consuming. Normalization can be used as a technique to represent the system considering resonant parameters instead of real circuit parameters, such as inductances, capacitances and resistances, which allows the attainment of generalized solutions [13]. This work deals with the mathematical formulation of the normalized analysis considering its fundamentals, the implication in the descriptive models and the circuit modeling for resonant, pulse-width modulation and high-efficiency DCDC converters. The main contributions of this work can be described as following:

- the fundamentals of the normalized analysis are explained in high-level of abstraction in order to show the influence of the main resonant parameters and the implication in the descriptive models;

- a mathematical formulation is used to formalize the concepts that are used in the methodology, such as: decomposing in resonant parameters, interdependence of resonant frequencies and equivalence transformation;

- analysis of the multi-stage Class D-E DC-DC converter in order to show the possibility of analysis without separating the rectifier and inverter subsystems.

In comparison with other methodologies, this work shows contributions because it does not resort to some simplifying assumptions, such as high loaded quality factor [14] and fundamental component approximation [15]-[17]. In addition, the normalized analysis provides analytical solutions that, in some cases, can be an useful tool to analyze complex systems and also can support design approaches based on circuit level simulations [18]. In the following, Section II shows the fundamentals of the normalized analysis. The mathematical formulation is performed in Section III. In Section IV, two circuits are analyzed in order to demonstrate the method. The design methodology is shown in Section V. Section VI deals with the experimental validation and some conclusions are drawn in Section VII. 


\section{FUNDAMENTALS OF NORMALIZED ANALYSIS}

This section shows the main concepts of the methodology under study. An overview of the system representation (II$A$ ), an explanation of the resonant parameters and its influence (II- $B$ ) and the implication in the descriptive models (II- $C$ ) are outlined in this section.

\section{A. Overview of the System Representation}

The main goal is system representation in a manner that does not require the use of circuit parameters, such as inductances, capacitances and resistances. Therefore, dimensionless parameters are used in order to represent a power converter considering the resonance among reactive components. In general, circuits are composed by resistances $R_{l}$, inductances $L_{m}$ capacitances $C_{n}$, operating angular frequency $\omega$ and input source $V_{i n}$ and $I_{i n}$, being $l, m$ and $n$, the indexes to define the number of components. The circuit parameters are decomposed in resonant parameters using the following rules [13]:

1. the number of extracted normalized resonant frequencies is the number of reactive components minus 1. Multiple capacitors in parallel or multiple inductors in series count as one reactive component;

2. the circuit load generates one quality factor, while each losses resistance generates an extra quality factor.;

3. the relation between the input source and the load defines the system's inverse transfer power ratio.

These concepts can be graphically explained in Figure 1. As it can be seen, the normalized resonant frequencies $A_{1}, A_{2}$ and $A_{3}$, the quality factors $Q_{1}$ and $Q_{2}$ and the inverse transfer power ratio $a$ are used to represent the system.

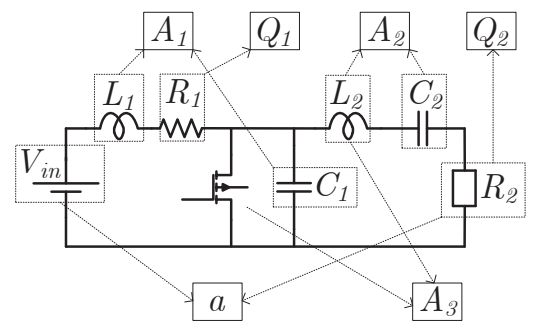

Fig. 1. Decomposing into resonant parameters scheme.

\section{B. Resonant Parameters}

The resonant parameters are: normalized resonant frequency $A_{j}$, quality factor $Q_{k}$ and the inverse transfer power ratio $a$, being $j$ and $k$ the indexes to define the number of resonant parameters. The normalized resonant frequency can be defined as follows:

$$
A_{j}=\frac{\omega_{j}}{\omega}
$$

Being, $\omega_{j}=\frac{1}{\sqrt{L_{m} C_{n}}}$ and $\omega$ the operating angular frequency. This parameter has influence on the converter waveforms by shaping them in a sinusoidal fashion. Furthermore, quantitatively, by increasing $A_{j}$, component values decrease, which can be used in circuit miniaturization. Nevertheless, components optimization requires more effort. In this case, the normalized analysis methodology is able to include optimum operation boundary conditions, as it is going to be shown in the next sections. Consider a general power converter that operates with one period of linearly increasing inductor current $i_{\text {in }}$, which ends at $T_{1}$. In addition, a second resonant period that starts in $T_{1}$, the influence of $A_{1}$ on the converter waveforms are shown in Figure 2 for the inductor current and in Figure 3 for output voltage. The quality factor can be defined as the ratio of the total electric energy stored in the circuit to the energy dissipate per cycle [19]. Nevertheless, considering its conceptual subjectivity, it can be advantageous to equate the quality factor as function of other circuit parameters. The quality factor can be equated for series and parallel output circuits as follows:

$$
\begin{gathered}
Q_{(\text {series })}=\frac{L_{m} \omega_{j}}{R_{l}}=\frac{1}{C_{n} \omega_{j} R_{l}} \\
Q_{(\text {parallel })}=\frac{R_{l}}{L_{m} \omega_{j}}=C_{n} \omega_{j} R_{l} .
\end{gathered}
$$

The inverse transfer power ratio $a$ is used as an interface between the input source $V_{\text {in }}$ and $I_{\text {in }}$ with the load $R$. In normalized analysis, it can be calculated as follows:

$$
\frac{1}{a}=\frac{1}{2 \pi} \int_{0}^{2 \pi}\left[\frac{v_{o}(\omega t)}{V_{i n}}\right]^{2} d \omega t .
$$
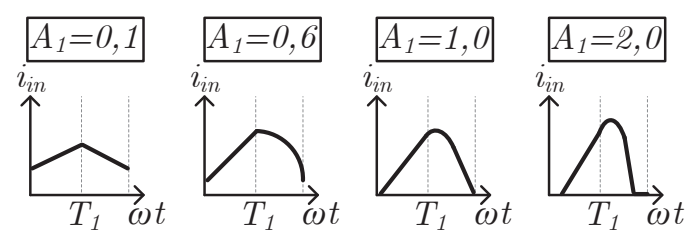

Fig. 2. Influence of $A_{1}$ on the current waveform.
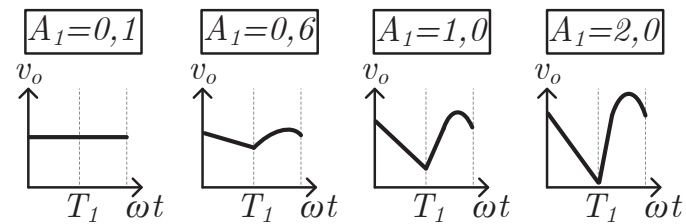

Fig. 3. Influence of $A_{1}$ on the voltage waveform.

Being $v_{o}(\omega t)$ the output voltage normalized by the input voltage $V_{i n}$, which can be dependent of $A_{j}, Q_{k}, a, \omega t$ and the initial conditions. The term $\frac{v_{o}(\omega t)}{V_{i n}}$ is a time-dependent equation obtained from the solution of the differential equations from each operating mode. In addition, the interface between $V_{\text {in }}, I_{\text {in }}$ and $R$ can be represented by:

$$
a=\frac{V_{\text {in }}}{R I_{\text {in }}} .
$$

In essence, the resonant parameters $A_{j}, Q_{k}$ and $a$ can be used to obtain a dimensionless mathematical model that is independent of circuit parameters, which has implications on the design methodology. 


\section{Implications in the Descriptive Models}

The aforementioned features of the resonance-based representation have implications for the descriptive models of the system design procedure. The main characteristic is that the real circuit parameters are omitted in exchange for more abstract parameters, which reduces the designer's perception of the converter. However, resonant parameters are suitable for parametric variation due to well-defined limit values. For example, in most of the cases, $A_{j}$ can be defined from 0.1 to 2.0 with steps of 0.1 for second/third order systems or 0.01 for high order systems, which gives a good understanding of the converter's behavior. It is because, the greater the number of reactive components, the more computational effort is required to solve the differential equations. Furthermore, in normalized analysis, circuit-level simulations are not required because the resonance-based representation leads to general time-domain normalized waveforms that are valid for any specification. As it can be seen in Figure 3, the output voltage waveforms are results from the normalized analysis for different values of $A_{j}$, which means that they are normalized by the input voltage and therefore are valid for any input specification.

\section{MATHEMATICAL FORMULATION}

In general, the normalized model can be described as a state-space model for each operating mode. The representation is shown as follows:

$$
\begin{aligned}
& \mathbf{x}_{\mathbf{e}}(\omega t)=\mathbf{E}_{\left.\lambda_{\left[A_{j}, Q_{k}\right.}\right]} \mathbf{x}_{\mathbf{e}}(\omega t)+\mathbf{F}_{\lambda} \\
& \mathbf{y}(\omega t)=\mathbf{G}_{\lambda_{\left[A_{j}, Q_{k}, a\right]}} \mathbf{x}_{\mathbf{e}}(\omega t)+\mathbf{H}_{\lambda} .
\end{aligned}
$$

It is considered the state matrix $\mathbf{E}_{\lambda}$, input matrix $\mathbf{F}_{\lambda}$, output matrix $\mathbf{G}_{\lambda}$ and transmission matrix $\mathbf{H}_{\lambda}$, where $\lambda$ represents the index for the converter operating mode. It can be seen that the input and transmission matrices are not multiplied by the input as in conventional state-space models, which means that the system is normalized by the input source. A vector space composed by terms that represent the square roots of the stored energy in the reactive components is used in an equivalence transformation that allows to convert the circuit parameters terms into resonant parameters. In this work, the normalized analysis is mathematically formulated.

Axiom 1. The number of extracted normalized resonant frequencies is the number of reactive components minus 1. Multiple capacitors in parallel or multiple inductors in series count as one reactive component.

Axiom 2. The circuit load generates one quality factor, while each losses resistance generates an extra quality factor.

In the decomposing in resonant parameters scheme, there are several possibilities for the choice of the pair formed by $L_{m}$ and $C_{n}$ in most of the cases, specially in third and higher order systems. However, any pair can be chosen, as formulated as follows:

Lemma 1. In the decomposing in resonant parameters, the resonant frequencies among reactive components are interdependent with each other.

Proof. Consider a fourth order system, which means that three resonant frequencies should be extracted:

$$
\omega_{1}=\frac{1}{\sqrt{L_{1} C_{1}}} ; \omega_{2}=\frac{1}{\sqrt{L_{2} C_{2}}} ; \omega_{3}=\frac{1}{\sqrt{L_{2} C_{1}}} .
$$

In addition, an extra frequency can be described as $\omega_{4}=$ $\frac{1}{\sqrt{L_{1} C_{2}}}$. Now, taking (8) into the equation for $\omega_{4}$ :

$$
\omega_{4}=\frac{1}{\sqrt{\frac{1}{\omega_{1}^{2} C_{1}} \frac{1}{\omega_{2}^{2} L_{2}}}}=\frac{1}{\sqrt{\frac{\omega_{3}^{2} L_{2}}{\omega_{1}^{2}} \frac{1}{\omega_{2}^{2} L_{2}}}}=\frac{1}{\sqrt{\frac{\omega_{3}^{2}}{\omega_{1}^{2} \omega_{2}^{2}}}} .
$$

And then:

$$
\omega_{4}=\frac{\omega_{1} \omega_{2}}{\omega_{3}}
$$

As a first example, consider the $L C R$ circuit in Figure 4. It is considered inductor $L_{1}$, capacitor $C_{1}$, resistance $R_{1}$, inductor current $i_{L_{1}}$ and capacitor voltage $v_{C_{1}}$.

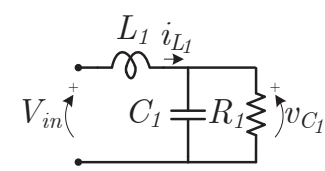

Fig. 4. $R L C$ circuit

In the normalization procedure, a state vector $\mathbf{x}(t)$ composed by the inductor current and the capacitor voltage, a new state vector composed by the square roots of the stored energy in the reactive components and a normalized output vector are defined by:

$$
\begin{gathered}
\mathbf{x}(t)=\left[\mathbf{i}_{\mathbf{L}_{\mathbf{1}}}, \mathbf{v}_{\mathbf{C}_{\mathbf{1}}}\right]^{T} \\
\mathbf{x}_{\mathbf{e}}(t)=\left[\mathbf{i}_{\mathbf{L}_{\mathbf{1}}} \frac{\sqrt{\mathbf{L}_{\mathbf{1}}}}{\sqrt{\mathbf{2}}}, \mathbf{v}_{\mathbf{C}_{\mathbf{1}}} \frac{\sqrt{\mathbf{C}_{\mathbf{1}}}}{\sqrt{\mathbf{2}}}\right]^{T} \\
\mathbf{y}(t)=\left[\frac{\mathbf{i}_{\mathbf{L}_{\mathbf{1}}}}{\mathbf{I}_{\mathbf{i n}}}, \frac{\mathbf{v}_{\mathbf{C}_{\mathbf{1}}}}{\mathbf{V}_{\mathbf{i n}}}\right]^{T} .
\end{gathered}
$$

Being, $I_{\text {in }}$ the input current and $V_{\text {in }}$ the input voltage. It can be seen that the output vector is normalized by the input source.

Axiom 3. The terms of the output vector are normalized by the input current or input voltage considering current variables or voltage variables, respectively.

Considering $\mathbf{x}(t)$ and $\mathbf{x}_{\mathbf{e}}(t)$ in their diagonal matrix form, an equivalence transformation $\mathbf{P}$ can be found by:

$$
\mathbf{P}=\mathbf{x}_{\mathbf{e}}(t) \mathbf{x}(t)^{-1}=\left(\begin{array}{cc}
\frac{\sqrt{L_{1}}}{\sqrt{2}} & 0 \\
0 & \frac{\sqrt{C_{1}}}{\sqrt{2}}
\end{array}\right) .
$$

Matrix $\mathbf{P}$ should be used to model the system considering the new state vector based on the square roots of the stored energy in the reactive components. First the system is represented 
considering the state vector $\mathbf{x}(t)$ and the output vector $\mathbf{y}(t)$ :

$$
\begin{gathered}
\dot{\mathbf{x}}(t)=\left(\begin{array}{cc}
0 & -\frac{1}{L_{1}} \\
\frac{1}{C_{1}} & -\frac{1}{R_{1} C_{1}}
\end{array}\right) \mathbf{x}(t)+\left(\begin{array}{c}
\frac{1}{L_{1}} \\
0
\end{array}\right) V_{i n} \\
\mathbf{y}(t)=\left(\begin{array}{cc}
\frac{1}{I_{i n}} & 0 \\
0 & \frac{1}{V_{i n}}
\end{array}\right) \mathbf{x}(t)+\left(\begin{array}{l}
0 \\
0
\end{array}\right) V_{i n} .
\end{gathered}
$$

Then, new state-space matrices are equated as follows:

$$
\dot{\mathbf{x}}_{\mathbf{e}}(\omega t)=\frac{1}{\omega} \mathbf{P}\left(\begin{array}{cc}
0 & -\frac{1}{L_{1}} \\
\frac{1}{C_{1}} & -\frac{1}{R_{1} C_{1}}
\end{array}\right) \mathbf{P}^{-1} \mathbf{x}_{\mathbf{e}}(\omega t)+\frac{1}{\omega} \mathbf{P}\left(\begin{array}{c}
\frac{1}{L_{1}} \\
0
\end{array}\right) V_{i n}
$$

Which results on:

$$
\dot{\mathbf{x}}_{\mathbf{e}}(\omega t)=\left(\begin{array}{cc}
0 & -\frac{1}{\sqrt{C_{1} L_{1}} \omega} \\
\frac{1}{\sqrt{C_{1} L_{1}} \omega} & -\frac{1}{R_{1} C_{1} \omega}
\end{array}\right) \mathbf{x}_{\mathbf{e}}(\omega t)+\left(\begin{array}{c}
\frac{1}{\sqrt{2 L_{1}} \omega} \\
0
\end{array}\right) V_{i n} .
$$

The term $1 / \omega$ in (17) indicates that the system is normalized by the operating angular frequency, which means that the independent variable, time $t$ was modified to $\omega t$.

Lemma 2. The multiplication of a scalar $\omega$ by the independent variable from the differential operator is considered a scalar by a vector multiplication, which maintains the function waveform.

Proof. Consider time $t$, which is the independent variable from the differential operator, as a vector and $x(t)$, any mathematical function. Multiplying $t$ by a scalar $\omega$, a new function, $x(\omega t)$ is obtained. Both $x(t)$ and $x(\omega t)$ have the same waveform fashion, even with modification in values of abscissa, which means that the function behavior is preserved.

In (18), the term $1 /\left(\sqrt{2 L_{1}} \omega\right)$ should be equal to 1 , which can be performed as follows:

$$
\mathbf{P}=\frac{\sqrt{2 L_{1}} \omega}{V_{\text {in }}} \mathbf{x}_{\mathbf{e}}(\omega t) \mathbf{x}(\omega t)^{-1} .
$$

This modification results on a state vector represented by:

$$
\mathbf{x}_{\mathbf{e}}(\omega t)=\left[\frac{\mathbf{L}_{\mathbf{1}} \omega}{\mathbf{V}_{\text {in }}}, \frac{\sqrt{\mathbf{C}_{\mathbf{1}}} \sqrt{\mathbf{L}_{\mathbf{1}}} \omega}{\mathbf{V}_{\text {in }}}\right]^{T} .
$$

It can be seen that $\mathbf{x}_{\mathbf{e}}(\omega t)$ appears being composed by constants. However, when solving the state-space equations, the states shown in (20) are fictitious representations that are solved as dynamical quantities that depend on $\omega t$.

Equating (17) considering (20):

$$
\dot{\mathbf{x}}_{\mathbf{e}}(\omega t)=\left(\begin{array}{cc}
0 & -\frac{1}{\sqrt{C_{1} L_{1} \omega}} \\
\frac{1}{\sqrt{C_{1} L_{1} \omega}} & -\frac{1}{R_{1} C_{1} \omega}
\end{array}\right) \mathbf{x}_{\mathbf{e}}(\omega t)+\left(\begin{array}{l}
1 \\
0
\end{array}\right) .
$$

The same procedure is used in (13):

$$
\mathbf{y}(\omega t)=\left(\begin{array}{cc}
\frac{1}{I_{i n}} & 0 \\
0 & \frac{1}{V_{i n}}
\end{array}\right) \mathbf{P}^{-1} \mathbf{x}_{\mathbf{e}}(\omega t)+\left(\begin{array}{l}
0 \\
0
\end{array}\right) V_{\text {in }}
$$

$$
\mathbf{y}(\omega t)=\left(\begin{array}{cc}
\frac{V_{i n}}{I_{i n} L_{1} \omega} & 0 \\
0 & \frac{1}{\sqrt{C_{1} L_{1}} \omega}
\end{array}\right) \mathbf{x}_{\mathbf{e}}(\omega t) .
$$

The mathematical model in (21) and (23) can be described as function of resonant parameters to a dimensionless model achievement.

Theorem 1. A generic system described by a statespace model $\mathbf{x}(t)=\mathbf{A} \mathbf{x}(t)+\mathbf{B} u$ and $\mathbf{y}(t)=\mathbf{C} \mathbf{x}(t)+\mathbf{D} u$ can be represented as a dimensionless model normalized by the input source and the operating frequency by using the transfer power ratio and the resonance among the reactive components.

Proof. Considering Axiom 1 and Axiom 2, a circuit can be interpreted by means of the resonance among reactive components and the circuit quality factor. Considering Lemma 2, a system can be normalized by the operating angular frequency. An equivalence transformation $\mathbf{P}$ can be used to modify the vector space of a system, also, it can be used to normalize the system in relation to the input source, as shown in (19), (20) and (21), which does not interfere in the state matrix. The circuit parameters used in (21) can be convert to resonant dimensionless parameters using (2), (3), (5) and (8):

- $\frac{1}{\sqrt{C_{1} L_{1}} \omega}=\frac{\omega_{1}}{\omega}=A_{1}$;

- $\frac{1}{R_{1} C_{1} \omega}=\frac{\omega_{1}}{R_{1} C_{1} \omega \omega_{1}}=\frac{A_{1}}{R_{1} C_{1} \omega_{1}}=\frac{A_{1}}{Q_{1}}$;

- $\quad \frac{V_{i n}}{I_{i n} L_{1} \omega}=\frac{R a}{L_{1} \omega}=\frac{R a \omega_{1}}{L_{1} \omega \omega_{1}}=Q_{1} A_{1} a$.

The system described in (21) and (23) can be represented in the format in (6) and (7):

$$
\begin{gathered}
\mathbf{E}_{I}=\left(\begin{array}{cc}
0 & -A_{1} \\
A_{1} & -\frac{A_{1}}{Q}
\end{array}\right) ; \mathbf{F}_{I}=\left(\begin{array}{l}
1 \\
0
\end{array}\right) \\
\mathbf{G}_{I}=\left(\begin{array}{cc}
Q_{1} A_{1} a & 0 \\
0 & -A_{1}
\end{array}\right) ; \mathbf{H}_{I}=\left(\begin{array}{l}
0 \\
0
\end{array}\right) .
\end{gathered}
$$

It is important to note that other relations are formed considering high order converters. Furthermore, each operating mode should be equated separately and solved considering initial and boundary conditions. The initial conditions are the initial inductor currents and initial capacitor voltages, $i_{L_{1}}(0) \ldots i_{L_{m}}(0)$ and $v_{C_{1}}(0) \ldots v_{C_{n}}(0)$. The normalized system in (6) for the first mode of the converter (mode $I$ ) returns $i_{L_{1}}(\omega t) \ldots i_{L_{m}}(\omega t)$ and $v_{C_{1}}(\omega t) \ldots v_{C_{n}}(\omega t)$, which are equations that depend on $A_{n a}, Q_{n q}$ and $\omega t$, in which, $n a$ goes from 1 to $n_{A_{j}}$ and $n q$ goes from 1 to $n_{Q_{k}}$, being $n_{A_{j}}$ and $n_{Q_{k}}$ the number of normalized resonant frequencies and the number of quality factors, respectively. The end of mode $I$ occurs in $\omega t$, then, it should be replaced by $t_{1}$. The equations from the solution of mode $I$ should be used as initial conditions for mode $I I$, described by $i_{L_{1}}\left(t_{1}\right) \ldots i_{L_{m}}\left(t_{1}\right)$ and $v_{C_{1}}\left(t_{1}\right) \ldots v_{C_{n}}\left(t_{1}\right)$. The solution of this system returns $i_{L_{1}}(\omega t) \ldots i_{L_{m}}(\omega t)$ and $v_{C_{1}}(\omega t) \ldots v_{C_{n}}(\omega t)$, which are equations that depend on $A_{n a}$, $Q_{n q}, \omega t, i_{L_{1}}(0) \ldots i_{L_{m}}(0)$ and $v_{C_{1}}(0) \ldots v_{C_{n}}(0)$. Again, $\omega t$ should be replaced by $t_{2}-t_{1}$, which identifies the duration of mode II. All operating modes should be solved. However, as long as the power converters are ruled by the duty cycle $D_{c}$, it should be used to replace $\omega t$ at the time that occurs the 
switch turn off. The initial conditions for the mode in which the switch turns off are identified by $i_{L_{1}}\left(D_{c} 2 \pi\right) \ldots i_{L_{m}}\left(D_{c} 2 \pi\right)$ and $v_{C_{1}}\left(D_{c} 2 \pi\right) \ldots v_{C_{n}}\left(D_{c} 2 \pi\right)$. For the last operating mode, the initial conditions are identified by $i_{L_{1}}\left(t_{\lambda-1}\right) \ldots i_{L_{m}}\left(t_{\lambda-1}\right)$ and $v_{C_{1}}\left(t_{\lambda-1}\right) \ldots v_{C_{n}}\left(t_{\lambda-1}\right)$. The solution of this system returns $i_{L_{1}}(2 \pi) \ldots i_{L_{m}}(2 \pi)$ and $v_{C_{1}}(2 \pi) \ldots v_{C_{n}}(2 \pi)$, which are equations that depend on $A_{n a}, Q_{n q}, t_{1} \ldots t_{\lambda_{-1}}, i_{L_{1}}(0) \ldots i_{L_{m}}(0)$, $v_{C_{1}}(0) \ldots v_{C_{n}}(0), i_{L_{1}}\left(t_{z}\right) \ldots i_{L_{m}}\left(t_{z}\right)$ and $v_{C_{1}}\left(t_{z}\right) \ldots v_{C_{n}}\left(t_{z}\right)$, in which $z$ goes from 1 to $\lambda-1$. The number of resulting variables $n_{v r}$ is described as follows:

$$
n_{v r}=n_{A_{j}}+n_{Q_{k}}+(\lambda-2)(n+m)+1 .
$$

The system in (6) is symbolically solved and it is used to solve (7) numerically. A parametric variation should be adopted, for example, duty cycle $D_{c}$ can be varied from 0.1 to 0.9 and the quality factors or the normalized resonant frequencies are defined in order to find the initial conditions. Considering the steady-state operation, the final conditions of the last operating mode should be equal to the initial conditions of the first operating mode. The linear system can be described in (27) at the top of the next page.

\section{CIRCUIT ANALYSIS}

\section{A. Resonant and PWM Converters}

As shown in Section II, the normalized resonant frequency $A_{j}$ influences the waveforms fashion. This concept can be applied directly in resonant converters analysis in order to obtain time-domain waveforms. In addition, it can be applied in PWM converters analysis. However, as $A_{j}$ increases, the waveforms for some operating mode can take sinusoidal fashion, which can be characterized as a resonant mode. In this subsection, a DCM (discontinuous conduction mode) Boost converter, shown in Figure 5, is analyzed, considering: inductor $L_{1}$, capacitor $C_{1}$, load $R_{1}$, switch $S$, diode $D$ and $i_{L_{1}}$, $v_{C_{1}}, i_{S}, v_{S}, i_{D}$ and $v_{D}$ the circuit variables. The DCM Boost converter has three operating modes: Mode $I\left(0<t \leq D_{c} 2 \pi\right)$ : $S$ on and $D$ off; Mode $I I\left(D_{c} 2 \pi<t \leq T_{2}\right)$ : $S$ off and $D$ on; Mode III $\left(T_{2}<t \leq 2 \pi\right)$ : $S$ off and $D$ off, being, $D_{c}$ the duty cycle and $T_{2}$ is the duration of mode $I I$. The normalized state-space model for the DCM Boost converter, considering the representation in (6) and (7), is described by: $\mathbf{x}_{\mathbf{e}}(t)=$ $\left[\frac{\mathbf{L}_{\mathbf{1}} \omega}{\mathbf{V}_{\text {in }}}, \frac{\sqrt{\mathbf{C}_{1}} \sqrt{\mathbf{L}_{1}} \omega}{\mathbf{V}_{\text {in }}}\right]^{T}, \mathbf{y}(t)=\left[\frac{i_{\mathbf{L}_{1}}}{\mathbf{I}_{\text {in }}}, \frac{\mathbf{i}_{\mathbf{S}}}{\mathbf{I}_{\text {in }}}, \frac{\mathbf{i}_{\mathbf{D}}}{\mathbf{I}_{\text {in }}}, \frac{\mathbf{v}_{\mathbf{C}_{\mathbf{1}}}}{\mathbf{V}_{\text {in }}}, \frac{\mathbf{v}_{\mathbf{S}}}{\mathbf{V}_{\text {in }}}, \frac{\mathbf{v}_{\mathbf{D}}}{\mathbf{V}_{\text {in }}}\right]^{T}$,

$$
\begin{aligned}
\mathbf{E}_{I} & =\left(\begin{array}{cc}
0 & 0 \\
0 & -\frac{A_{1}}{Q_{1}}
\end{array}\right), \mathbf{F}_{I}=\left(\begin{array}{l}
1 \\
0
\end{array}\right), \mathbf{E}_{I I}=\left(\begin{array}{cc}
0 & -A_{1} \\
A_{1} & -\frac{A_{1}}{Q_{1}}
\end{array}\right), \\
\mathbf{F}_{I I} & =\left(\begin{array}{l}
1 \\
0
\end{array}\right), \mathbf{E}_{I I I}=\left(\begin{array}{cc}
0 & 0 \\
0 & -\frac{A_{1}}{Q_{1}}
\end{array}\right), \mathbf{F}_{I I I}=\left(\begin{array}{l}
0 \\
0
\end{array}\right), \\
\mathbf{G}_{I} & =\left(\begin{array}{cc}
a Q_{1} A_{1} & 0 \\
a Q_{1} A_{1} & 0 \\
0 & 0 \\
0 & A_{1} \\
0 & 0 \\
0 & -A_{1}
\end{array}\right), \mathbf{G}_{I I}=\left(\begin{array}{cc}
a Q_{1} A_{1} & 0 \\
0 & 0 \\
a Q_{1} A_{1} & -a A_{1} \\
0 & A_{1} \\
0 & 0 \\
0 & -A_{1}
\end{array}\right),
\end{aligned}
$$

$$
\mathbf{G}_{I I I}=\left(\begin{array}{cc}
a Q_{1} A_{1} & 0 \\
0 & 0 \\
0 & 0 \\
0 & A_{1} \\
0 & 0 \\
0 & -A_{1}
\end{array}\right), \mathbf{H}_{I, I I}=\left(\begin{array}{l}
0 \\
0 \\
0 \\
0 \\
0 \\
0
\end{array}\right), \mathbf{H}_{I I I}=\left(\begin{array}{l}
0 \\
0 \\
0 \\
0 \\
1 \\
1
\end{array}\right) .
$$

The normalized state equations for the first operating mode should be solved considering the initial conditions for inductor current and capacitor voltage, $i_{L_{1}}(0)$ and $v_{C_{1}}(0)$. The solution of this mode is used as initial conditions for the second mode. The system to be solved is described as follows:

$$
\left(\begin{array}{l}
i_{L_{1}}(2 \pi)_{\left[A_{1}, Q_{1}, D_{c}, i_{L_{1}}(0), v_{C_{1}}(0)\right]} \\
v_{C_{1}}(2 \pi)_{\left[A_{1}, Q_{1}, D_{c}, i_{L_{1}}(0), v_{C_{1}}(0)\right]}
\end{array}\right)=\left(\begin{array}{l}
i_{L_{1}}(2 \pi) \\
v_{C_{1}}(2 \pi)
\end{array}\right)
$$

By sweeping $D_{c}$ and defining $Q_{1}$ and $A_{1}$, the initial conditions can be found for any operating point by using a numerical method. Then, the output equations should be solved in order to obtain normalized time-domain steady state waveforms for the converter. Considering $D_{c}=0.25$, the normalized waveforms for the DCM Boost converter are shown in Figure 6 for $Q_{1}=25$ and $A_{1}=0.1$ and in Figure 7 for $Q_{1}=25$ and $A_{1}=1.8$.

\section{B. High-Efficiency DC-DC Resonant Converters}

A DC-DC resonant converter can be obtained by cascading a resonant inverter and a resonant rectifier. An example is the Class D-E converter that has some of its first appearances in literature in [19], [20]. The Class D-E converter is shown in Figure 8, in which, $V_{\text {in }}$ is the input source; $L_{1}, L_{2}$ are the inductors; $C_{1}, C_{2}$ and $C_{3}$ are the capacitors; $S_{1}$ and $S_{2}$ are the switches; $D$ is the rectifier diode; $R_{1}$ is the load and $i_{S_{1}}, i_{S_{2}}$, $i_{L_{1}}, i_{L_{2}}, i_{D}, i_{o}, v_{S_{1}}, v_{S_{2}}, v_{C_{1}}, v_{D}$ and $v_{o}$ are the circuit variables. This converter has four operating modes: Mode $I\left(0<t \leq T_{1}\right)$ : $S_{1}$ on, $S_{2}$ off and $D$ off; Mode $I I\left(T_{1}<t \leq D_{c} 2 \pi\right): S_{1}$ on, $S_{2}$ off and $D$ on; Mode III $\left(D_{c} 2 \pi<t \leq T_{2}\right): S_{1}$ off, $S_{2}$ on and $D$ on; Mode $I V\left(T_{2}<t \leq 2 \pi\right): S_{1}$ off, $S_{2}$ on and $D$ off, being $T_{1}$ and $T_{2}$ the durations of Mode $I$ and $I I I$, respectively.

The converter has five reactive components, which leads to four resonant frequencies that can be described by: $\omega_{1}=$ $\frac{1}{\sqrt{L_{1} C_{1}}}, \quad \omega_{2}=\frac{1}{\sqrt{L_{2} C_{1}}}, \quad \omega_{3}=\frac{1}{\sqrt{L_{2} C_{2}}}$ and $\omega_{4}=\frac{1}{\sqrt{L_{1} C_{3}}}$. The normalized resonant frequencies are: $A_{1}=\frac{\omega_{1}}{\omega}, A_{2}=\frac{\omega_{2}}{\omega}$, $A_{3}=\frac{\omega_{3}}{\omega}$ and $A_{4}=\frac{\omega_{4}}{\omega}$. The circuit quality factor can be used as function of $\omega_{1}: Q_{1}=\frac{\omega_{1} L_{1}}{R_{L}}=\frac{1}{\omega_{1} C_{1} R_{L}}$. The system can be represented by (6) and (7). However, in this case, the system to be solved needs some optimization considerations to improve its operation. The state vector is equated by: $\mathbf{x}_{\mathbf{e}}(t)=\left[\frac{\mathbf{L}_{\mathbf{1}} \omega}{\mathbf{V}_{\text {in }}}, \frac{\sqrt{\mathbf{L}_{\mathbf{1}} \mathbf{L}_{\mathbf{2}}} \omega}{\mathbf{V}_{\text {in }}}, \frac{\sqrt{\mathbf{C}_{\mathbf{1}} \mathbf{L}_{\mathbf{1}}} \omega}{\mathbf{V}_{\text {in }}}, \frac{\sqrt{\mathbf{C}_{\mathbf{2}} \mathbf{L}_{\mathbf{1}}} \omega}{\mathbf{V}_{\text {in }}}, \frac{\sqrt{\mathbf{C}_{\mathbf{3}} \mathbf{L}_{\mathbf{1}}} \omega}{\mathbf{V}_{\text {in }}}\right]^{T}$.

At the diode $D$ turn-off, $i_{C_{2}}$ is zero, which allows a soft turn-off condition. The diode $D$ turns off at zero $d v / d t$, which can be used as a condition for the system solver described as $\left(A_{1} / A_{2}\right) i_{L_{1}}\left(T_{2}\right)=i_{L_{2}}\left(T_{2}\right)$. The term $\left(A_{1} / A_{2}\right)$ is necessary because the states are described in a normalized state-space model. In addition, the diode turns on at low $d v / d t$. In this case, the condition is $v_{C_{2}}(T 1)=0$. By using the methodology, the normalized time-domain steady state waveforms can be found. As aforementioned, the advantage of the normalized 


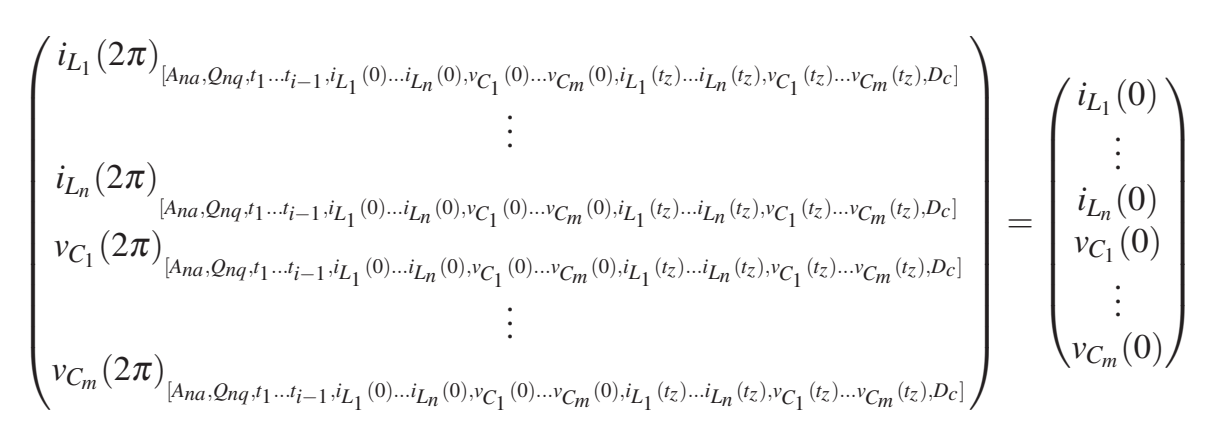

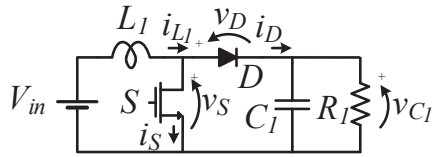

Fig. 5. Boost converter.

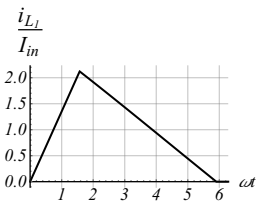

(a)

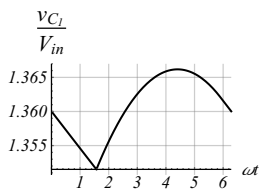

(d)

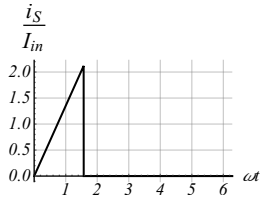

(b)

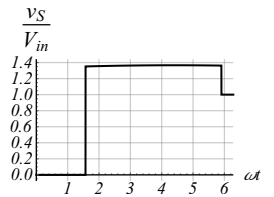

(e)

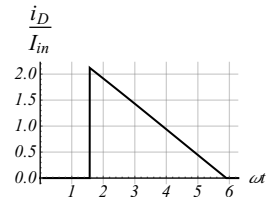

(c)

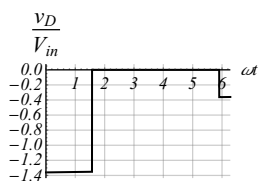

(f)

Fig. 6. Normalized waveforms for the DCM Boost converter considering $A_{1}=0.1$. (a) Inductor $L_{1}$ current. (b) Switch $S$ current. (c) Diode $D$ current. (d) Capacitor $C_{1}$ voltage. (e) Switch $S$ voltage. (f) Diode $D$ voltage.

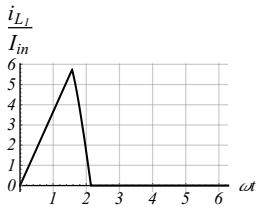

(a)

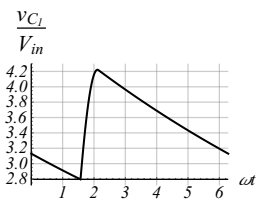

(d)

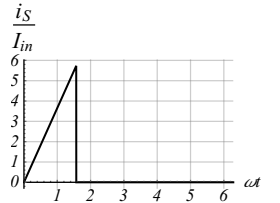

(b)

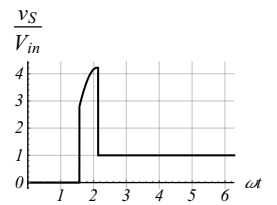

(e)

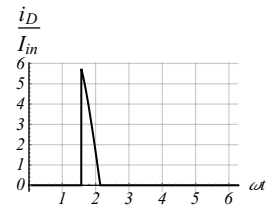

(c)

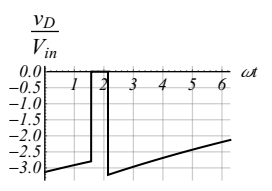

(f)

Fig. 7. Normalized waveforms for the DCM Boost converter considering $A_{1}=1.8$. (a) Inductor $L_{1}$ current. (b) Switch $S$ current. (c) Diode $D$ current. (d) Capacitor $C_{1}$ voltage. (e) Switch $S$ voltage. (f) Diode $D$ voltage.

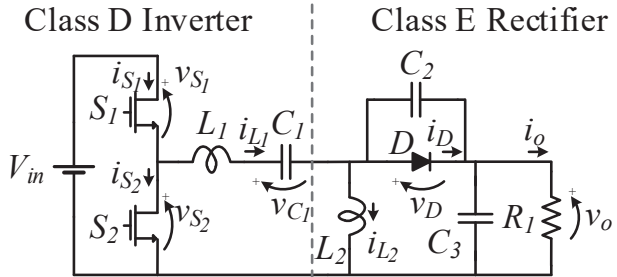

Fig. 8. Class D-E resonant DC-DC converter.

model is that the system can be analyzed without resorting to simplifying assumptions, such as high loaded quality factor and fundamental component sinusoidal approximation. The normalized waveforms for $D_{c}=0.5, A_{1}=0.87, A_{2}=0.09$, $A_{3}=0.11$ and $A_{4}=0.01$ are shown in Figure 9 for $Q_{1}=10$ and in Figure 10 for $Q_{1}=2$.

The normalized analysis allows the attainment of gain and component stress curves for any operating point by sweeping $D_{c}, Q_{1}$ and the normalized resonant frequencies. A parametric sweep on $Q_{1}$ was performed for $D_{c}=0.5$ and a set of $A_{1}=\{0.3,0.5,0.8\}$. Also, a parametric sweep on $D_{c}$ was performed for $Q_{1}=10$. The main gain and component stress curves are shown in Figure 11. It can been seen in Figure 11.a that the transfer power ratio $T_{P O T}$ is higher for $A_{1}=0.8$ because it is closer to the resonance frequency. The switch $S_{1}$ peak current and diode $D$ peak current are shown in Figure 11.b and Figure 11.c respectively. The output voltage ripple $\Delta V_{o}$ as function of $D_{c}$ is shown in Figure 11.d. The transfer power ratio, diode turn-on time, diode turnoff time and capacity $C_{p}$, which is defined as the inverse of the multiplication of current and voltage peak values, as function of $Q_{1}$ are shown in Figure 11.e-h, respectively. These curves can be used to analyze the converter behavior without resorting to circuit-level simulations. Furthermore, the results can be stored in a database and used to design the real circuit parameters based on the resonant parameters.

\section{DESIGN METHODOLOGY}

Equations 1,2 and 3 are used to derive the design equations. For the Class D-E converter, the reactive components can be designed by:

$$
\begin{gathered}
L_{1}=\frac{Q_{1} R_{1}}{A_{1} \omega} ; L_{2}=\frac{Q_{1} A_{1} R_{1}}{A_{2}^{2} \omega} \\
C_{1}=\frac{1}{Q_{1} A_{1} R_{1} \omega} ; C_{2}=\frac{A_{2}^{2}}{Q_{1} A_{3}^{2} A_{1} R_{1} \omega} ; C_{3}=\frac{A_{1}}{Q_{1} A_{4}^{2} R_{1} \omega} .
\end{gathered}
$$




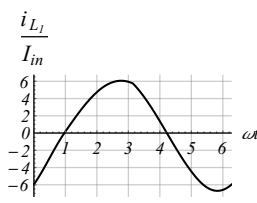

(a)

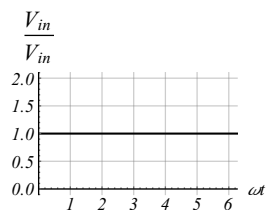

(g)

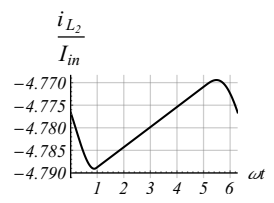

(b)

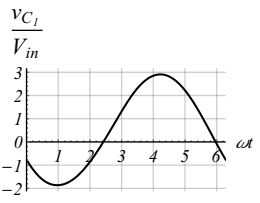

(h)

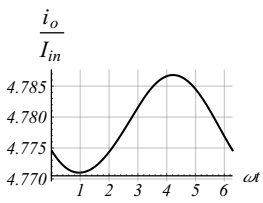

(c)

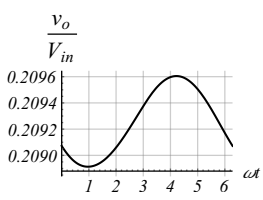

(i)

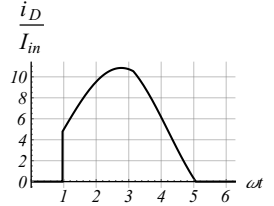

(d)

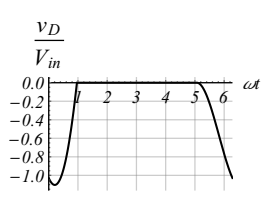

(j)

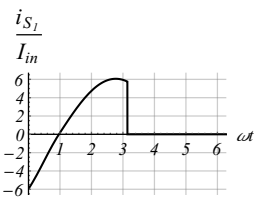

(e)

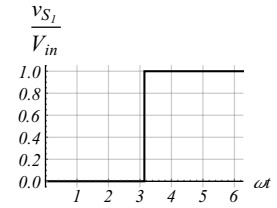

(k)

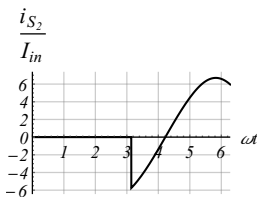

(f)

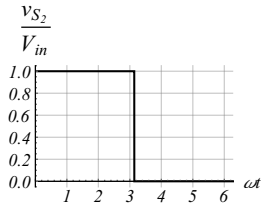

(1)

Fig. 9. Normalized waveforms for the Class D-E converter considering $Q_{1}=10$. (a) Inductor $L_{1}$ current. (b) Inductor $L_{2}$ current. (c) Output current $i_{o}$. (d) Diode $D$ current. (e) Switch $S_{1}$ current. (f) Switch $S_{2}$ current. (g) Input voltage. (h) Capacitor $C_{1}$ voltage. (i) Output voltage (j) Diode $D$ voltage. (k) Switch $S_{1}$ voltage. (1) Switch $S_{2}$ voltage.

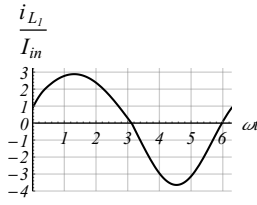

(a)

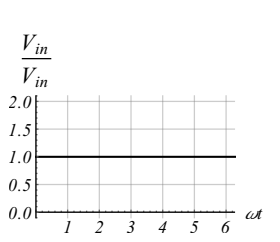

(g)

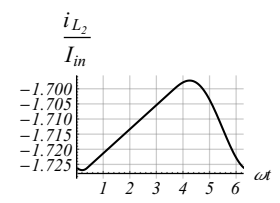

(b)

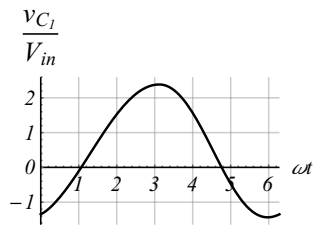

(h)

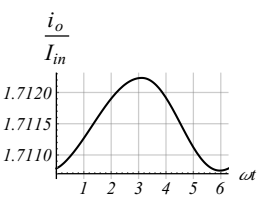

(c)

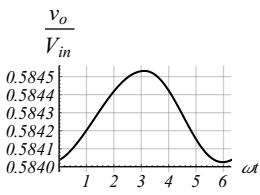

(i)

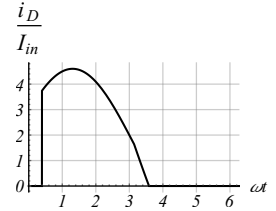

(d)

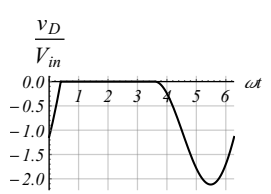

(j)

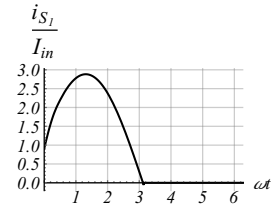

(e)

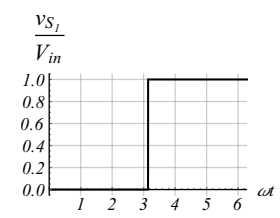

(k)

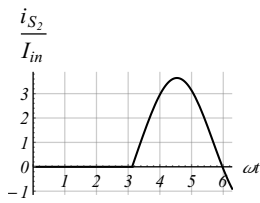

(f)

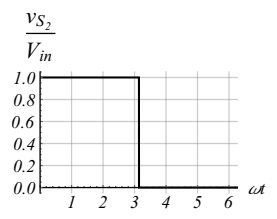

(1)

Fig. 10. Normalized waveforms for the Class D-E converter considering $Q_{1}=2$. (a) Inductor $L_{1}$ current. (b) Inductor $L_{2}$ current. (c) Output current $i_{o}$. (d) Diode $D$ current. (e) Switch $S_{1}$ current. (f) Switch $S_{2}$ current. (g) Input voltage. (h) Capacitor $C_{1}$ voltage. (i) Output voltage (j) Diode $D$ voltage. (k) Switch $S_{1}$ voltage. (1) Switch $S_{2}$ voltage.

There are several possibilities to design the converter. First, some specifications should be defined, such as input voltage $V_{i n}$, output power $P_{o}$ and operating frequency $f$.

Considering the results for any operating point that are obtained with the normalized analysis, the resonant parameters, $A_{1}, A_{2}, A_{3}, A_{4}$ and $Q_{1}$ are used regarding a selected duty cycle $D_{c}$ or transfer power ratio $T_{P O T}$. The relation $V_{o}=\sqrt{T_{P O T}} V_{i n}$ is used as the interface between the output voltage $V_{o}$ and $V_{i n}$.

In conventional design methodologies, the output ripple is used to design the output capacitor. In the present work, for the Class D-E converter, $A_{4}$ is the main parameter used to design the output capacitor. In this case, reducing the value of $A_{4}$ increases the value of $C_{3}$ and reduces the output ripple. However, as long as the proposed methodology obtains normalized waveforms previously, as shown in Figure 9 and Figure 10, the designer is able to select the output ripple based on them, ensuring that they are valid for any input and operating frequency. A design example is going to be considered. By selecting $D_{c}=0.5$ and $Q_{1}=10$, the resonant parameters are $A_{1}=0.87, A_{2}=0.09, A_{3}=0.11, A_{4}=0.01$ and $T_{P O T}=0.044$. The design specifications and parameters are shown in Table I.

In order to obtain the dimensional values, the peak values from the normalized waveforms should be multiplied by $V_{\text {in }}$ or $I_{\text {in }}$ for voltage or current circuit variables, respectively. As an example, the inductor $L_{1}$ current normalized peak value in Figure 9.a is 6.06. Therefore, $6.06 \times I_{\text {in }}=6.06 \times 0.05=$ $0.303 A$. The same procedure was performed for the other circuit variables and validated by SPICE simulations. This dimensional analysis in summarized in Table II. 


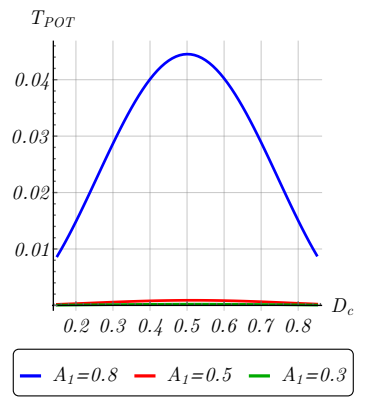

(a)

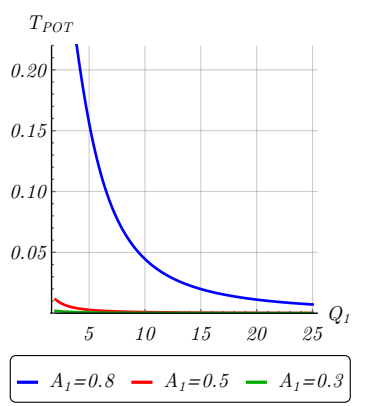

(e)

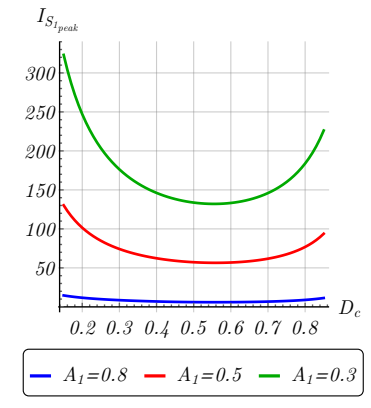

(b)

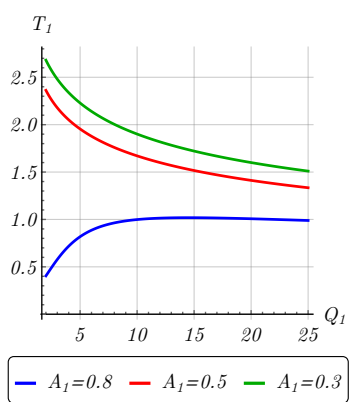

(f)

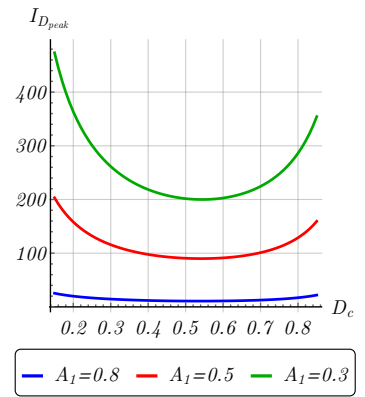

(c)

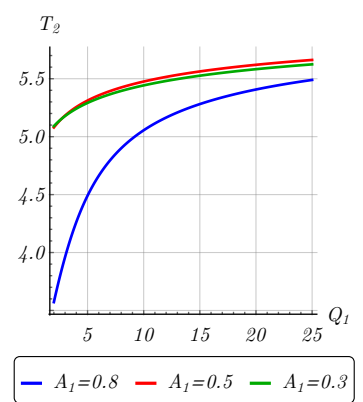

(g)

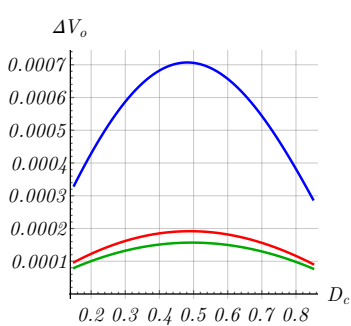

- $A_{1}=0.8-A_{1}=0.5-A_{1}=0.3$

(d)

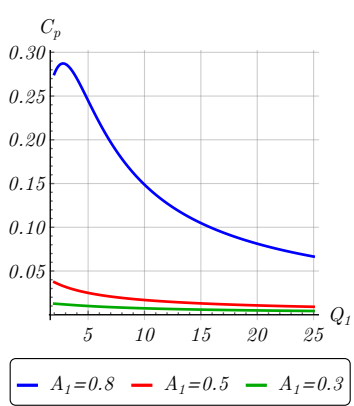

(h)

Fig. 11. Normalized gain and component stress curves for the Class D-E converter. (a) Transfer power ratio $T_{P O T}$ as function of $D_{c}$ for $Q_{1}=10$. (b) Switch $S_{1}$ peak current as function of $D_{c}$ for $Q_{1}=10$. (c) Diode $D$ peak current as function of $D_{c}$ for $Q_{1}=10$. (d) Output voltage ripple $\Delta V_{o}$ as function of $D_{c}$ for $Q_{1}=10$. (e) Output current ripple $\Delta I_{o}$ as function of $D_{c}$ for $Q_{1}=10$. (f) Transfer power ratio $T_{P O T}$ as function of $Q_{1}$. (g) Diode turn-on time $T_{1}$ as function of $Q_{1}$. (h) Diode turn-off time $T_{2}$ as function of $Q_{1}$. (i) Capacity $C_{p}$ considering switch $S_{2}$ as function of $Q_{1}$.

TABLE I

Design Example

\begin{tabular}{|c|c|}
\hline \multicolumn{2}{|c|}{ Design Specifications and Parameters } \\
\hline Specification/Parameter & Value \\
\hline Input voltage, $V_{i n}$ & $55 \mathrm{~V}$ \\
\hline Output power, $P_{o}$ & $3 W$ \\
\hline Output voltage, $V_{o}$ & $11.6 \mathrm{~V}$ \\
\hline Operating frequency, $f$ & $500 \mathrm{kHz}$ \\
\hline Duty cycle, $D_{c}$ & 0.5 \\
\hline Quality factor, $Q_{1}$ & 10 \\
\hline Normalized resonant frequency, $A_{1}$ & 0.87 \\
\hline Normalized resonant frequency, $A_{2}$ & 0.09 \\
\hline Normalized resonant frequency, $A_{3}$ & 0.11 \\
\hline Normalized resonant frequency, $A_{4}$ & 0.01 \\
\hline Resonant inductor, $L_{1}$ & $186 \mu H$ \\
\hline Rectifier inductor, $L_{2}$ & $14 \mathrm{mH}$ \\
\hline Resonant capacitor, $C_{1}$ & $820 p F$ \\
\hline Rectifier capacitor, $C_{2}$ & $560 p F$ \\
\hline Output capacitor, $C_{3}$ & $560 \mu F$ \\
\hline Load, $R_{1}$ & $51 \Omega$ \\
\hline
\end{tabular}

\section{EXPERIMENTAL VALIDATION}

In order to validate the theoretical approach, the Class DE DC-DC converter designed in Section V was implemented. For the experimental validation, the specifications and design parameters are shown in Table I. The diode voltage is shown in Figure 12, in which the zero-derivative switching can be observed. The switches $S_{1}$ and $S_{2}$ voltages are shown in Figure 13. The resonant current $i_{L_{1}}$ and resonant voltage $v_{S_{2}}$ are shown in Figure 14. One can see that the resonant current is lagged in relation to the resonant voltage
TABLE II

Dimensional Analysis

\begin{tabular}{|c|c|c|c|}
\hline \multicolumn{4}{|c|}{$V_{\text {in }}=55 \mathrm{~V} I_{\text {in }}=0.05$} \\
\hline $\begin{array}{c}\text { Circuit } \\
\text { variable }\end{array}$ & $\begin{array}{c}\text { Normalized } \\
\text { value }\end{array}$ & $\begin{array}{c}\text { Dimensional } \\
\text { value }\end{array}$ & $\begin{array}{c}\text { SPICE } \\
\text { simulation }\end{array}$ \\
\hline Inductor $L_{1}$ current, $i_{L_{1}}$ & 6.06 & $0.303 A$ & $0.304 A$ \\
\hline Inductor $L_{2}$ current, $i_{L_{2}}$ & -4.772 & $-0.2386 A$ & $-0.2386 A$ \\
\hline Diode $D$ current, $i_{D}$ & 10.84 & $0.542 A$ & $0.53 A$ \\
\hline Output voltage $V_{o}$ & 0.2092 & $11.506 \mathrm{~V}$ & $11.6 \mathrm{~V}$ \\
\hline Diode $v_{D}$ voltage, $v_{D}$ & -1.17 & $-64.35 \mathrm{~V}$ & $-61.9 \mathrm{~V}$ \\
\hline Switch $S_{1}$ voltage, $v_{S_{1}}$ & 1 & $55 \mathrm{~V}$ & $55 \mathrm{~V}$ \\
\hline Switch $S_{2}$ voltage, $v_{S_{2}}$ & 1 & $55 \mathrm{~V}$ & $55 \mathrm{~V}$ \\
\hline Capacitor $C_{1}$ voltage, $v_{C_{1}}$ & 2.7 & $148.5 \mathrm{~V}$ & $143.02 \mathrm{~V}$ \\
\hline
\end{tabular}

that becomes zero before the current becomes zero, which ensures zero-voltage switching. Finally, the output voltage is shown in Figure 15. When operating in high frequencies, the switches capacitances play a role and they can be included in the theoretical analysis. Although, including extra reactive components leads to extra normalized resonant frequencies. Therefore, higher computational effort is required in order to achieve more accurate results when solving the normalized state-space equations.

\section{CONCLUSION}

This paper has presented a formalization of an analysis methodology for power electronic converters based on the system representation by resonant parameters. The fundamentals of the normalized analysis, a mathematical formulation and circuit analysis for the DCM Boost converter 


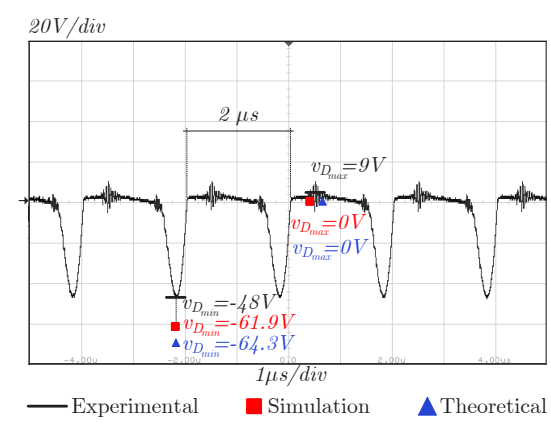

Fig. 12. Experimental result for diode $D$ voltage, $v_{D}$ (20 V/div; $1 \mu$ s/div).

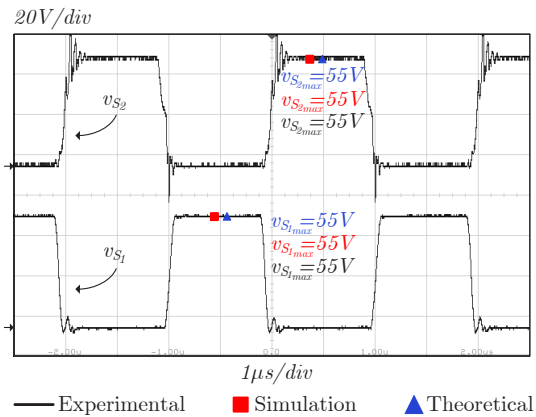

Fig. 13. Experimental result for switches $S_{1}$ and 2 voltages, $v_{S_{1}}$ and $v_{S_{2}}(20 \mathrm{~V} / \mathrm{div} ; 1 \mu \mathrm{s} / \mathrm{div})$.

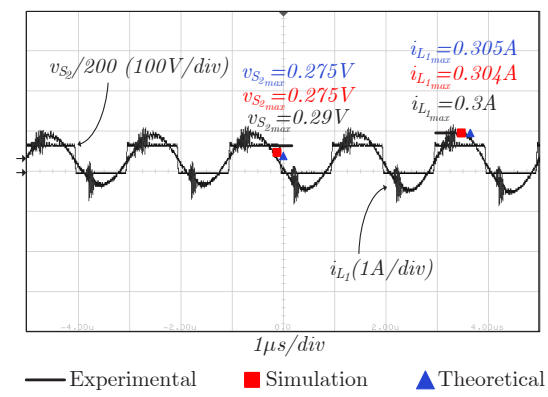

Fig. 14. Experimental result for resonant current, $i_{L_{1}}$ and resonant voltage, $v_{S_{2}}(1 \mathrm{~A} / \mathrm{div}, 100 \mathrm{~V} / \mathrm{div} ; 1 \mu \mathrm{s} / \mathrm{div})$.

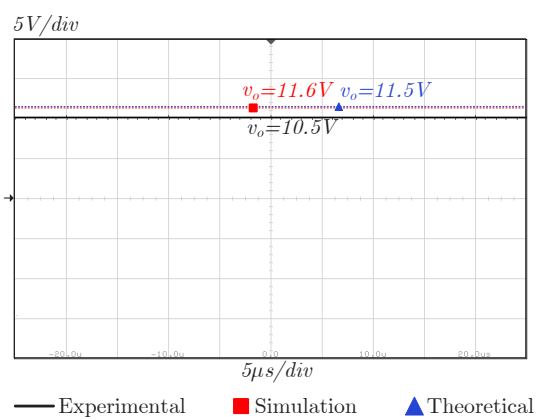

Fig. 15. Experimental result for output voltage, $v_{o}(5 \mathrm{~V} / \mathrm{div} ; 5 \mu \mathrm{s} / \mathrm{div})$. and the Class D-E converter were explored in order to show the theoretical development. The main advantage of the normalized analysis is that the system can be represented by dimensionless resonant parameters, which allows the obtainment of generalized time-domain waveforms for any operating point and specifications. The contributions of this paper are the mathematical formalization of the normalization procedure and the demonstration of the multi-stage Class D$\mathrm{E}$ resonant converter, which is analyzed without separating the inverter from the rectifier stage and without resorting to some simplifying assumptions, such as, high loaded quality factor and sinusoidal approximation. Experimental results for the Class D-E converter are shown to validate the theoretical approach. The results can be used to design high efficiency power converters that are ubiquitous in several applications, such as, battery charges, lighting systems and wireless power transfer.

\section{ACKNOWLEDGEMENTS}

This study was financed in part by the Coordenação de Aperfeiçoamento de Pessoal de Nível Superior - Brasil (CAPES/PROEX) - Finance Code 001. This work was supported by the National Council for Scientific and Technological Development (CNPq).

\section{REFERENCES}

[1] M. Liu, Y. Qiao, S. Liu, C. Ma, "Analysis and Design of A Robust Class $E^{2}$ DC-DC converter for Megahertz Wireless Power Transfer", IEEE Trans. Power Electron., vol. 32, pp. 2835-2845, May. 2016.

[2] R. C. Fernandes, A. A. De Oliveira, "Comparative Performance Evaluation of Magnetic Couplers for Wireless Power Transfer Applications", IEEE 13th Brazilian Power Electronics Conference and 1st Southern Power Electronics Conference (COBEP/SPEC), pp. 1-6, 2015.

[3] L. Zhao, D. J. Thrimawithana, U. K. Madawala, C. A. Baguley, "Performance Optimization of LC Bi-Directional Inductive Power Transfer System", IEEE 13th Brazilian Power Electronics Conference and 1st Southern Power Electronics Conference (COBEP/SPEC), pp. 1-6, 2015.

[4] N. F. O. Da Silva, M. L. Heldwein, A. J. Perin, "Conversor Wireless com Retificador ZCS", Revista Eletrônica de Potência, vol. 22, no. 3, pp. 298-309, 2017.

[5] F. Meddour, Z. Dibi, "An Efficient Small Size Electromagnetic Energy Harvesting Sensor for LowDC-power Applications", IET Microwaves, Antennas \& Propagation, vol. 11, pp. 483-489, Apr. 2017.

[6] D. R. Rodrigues, "A MCU based DC-DC Step-Up Converter Providing Regulation of its Own Power Supply", IEEE 13th Brazilian Power Electronics Conference and 1st Southern Power Electronics Conference (COBEP/SPEC), pp. 1-4, 2015.

[7] T. C. Naidon, L. S. Mendonça, F. E. Bisogno "A Normalized Approach for Analysis and Design a Flyback Resonant Converter for Energy Harvesting 
Systems", Brazilian Power Electronics Conference (COBEP), pp. 1-6, 2017.

[8] Y. Wang, X. Deng, Y. Wang, D. Xu, "SingleStage Bridgeless LED Driver Based on a CLCL Resonant Converter", IEEE Transactions on Industry Applications, vol. 54, pp. 1832-1841, Nov. 2017.

[9] P. E. Bolzan, I. B. Barboza, J. L. Putzke, V. L. Rosa, R. N. Do Prado, "Switched Capacitor Converter with Variable Duty Cycle to Feed LED Tubular Lamp", Brazilian Power Electronics Conference (COBEP), pp. 1-5, 2017.

[10] D. C. Pereira, P. L. Tavares, P. S. Almeida, G. M. Soares, F. L. Tofoli, H. A. C. Braga, "Improved Photoelectrothermal Model with Thermal Parameters Variation Applied to an Extra-High Current COB LED", Revista Eletrônica de Potência, 2019.

[11] C. R. B. S. Rodrigues, P. S. Alem, P. S. Almeida, G. M. Soares, M. F. Braga, H. A. C. Braga, "A Novel Linear Circuit for Current Equalization In LED Strings", Revista Eletrônica de Potência, vol. 18, no. 3, 2013.

[12] Z. Zhang, J. Lin, Y. Zhou, X. Ren, "Analysis and Decoupling Design of a $30 \mathrm{Mhz}$ Resonant Sepic Converter", IEEE Trans. Power Electron., vol. 31, pp. 4536-4548, Jun. 2016.

[13] F. E. Bisogno, "Energy-related System Normalization and Decomposition Targeting Sensitivity Consideration", PhD Dissertation, 2007.

[14] T. Nagashima, X. Wei, E. Bou, E. Alarcón, M. K. Kazimierczuk, H. Sekiya, "Analysis and Design of Loosely Inductive Coupled Wireless Power Transfer System Based on Class- $E^{2}$ DC-DC Converter for Efficiency Enhancement", IEEE Trans. on Circuit and Systems I: Regular Papers, vol. 62, pp. 2781-2791, Nov. 2015.

[15] S. Yu, "A New Compact and High Efficiency Resonant Converter", IEEE Applied Power Electronics Conference and Exposition (APEC), Mar. 2016.
[16] M. T. Outeiro, G. Buja, D. Czarkowski, "Resonant Power Converters: An Overview with Multiple Elements in the Resonant Tank Network", IEEE Ind. Electron. Magazine., vol. 10, pp. 21-45, Jun. 2016.

[17] Y. Guan, Y. Wang, W. Wang, D. Xu, "Analysis and Design of a High-Frequency DC/DC Converter Based on a Resonant Rectifier", IEEE Trans. Ind. Electronics, vol. 64, pp. 8492-8503, Apr. 2017.

[18] K. Watanabe, M. Abe, T. Sai, Y. Sugimoto, "A Fast and Precise Circuit Simulation Method for Switching Power Converters using a Mixture of Circuits and Behavioral Models", IEEE Asia Pacific Conference on Circuit and Systems., Nov. 2014.

[19] M. K. Kazimierczuk, D. Czarkowski, "Resonant Power Converters", John Wiley \& Sons, Inc., 1995.

[20] M. K. Kazimierczuk, W. Szaraniec, "Class DE Resonant DC/DC Converter", IEEE Trans. on Aerospace and Electronic Systems, vol. 29, pp. 963-976, Jul. 1993.

\section{BIOGRAPHIES}

Lucas S. Mendonça, born in 1993 in Santa Maria-Brazil, is a control and automation engineer (2015) and master in Electrical Engineer (2017) with the Universidade Federal de Santa Maria, Brazil. He is currently a doctorate researcher in Fraunhofer IIS/EAS, Germany. His areas of interest are: power electronics, resonant power conversion and contactless energy transfer systems.

Fábio E. Bisogno, born in 1973 in Santa Maria-Brazil is an electrical engineer (1999), master (2001) with the Universidade Federal de Santa Maria and Dr.-Ing. in Electrical Engineer (2006) with the Technische Universität Chemnitz, Germany. He is currently a full professor at Universidade Federal de Santa Maria. His areas of interest are: resonant converters, self-oscillating systems and sensitivity analysis. 